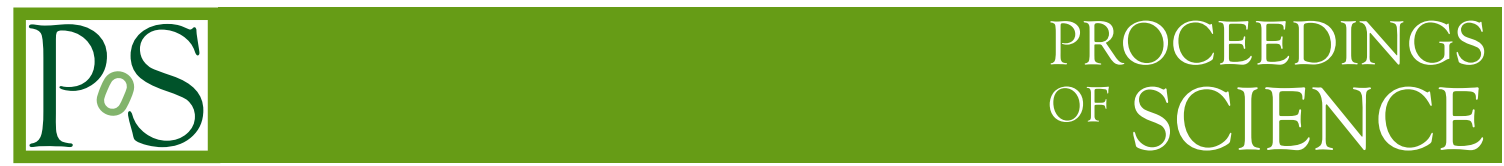

\title{
New results from $Y(4260)$ decays at BESIII
}

\author{
Ke LI (for BESIII Collaboration)* \\ Institute of high energy physics, Beijing 100049, People's Republic of China \\ E-mail: likelihep.ac.cn
}

A series study of $Y(4260)$ are performed with the world's largest data sample around $\sqrt{s}=4.26$ $\mathrm{GeV}$ at BESIII, similar structures around $4.22 \mathrm{GeV}$, denoted as $Y(4220)$, are observed in the cross section distributions for $e^{+} e^{-} \rightarrow \pi^{+} \pi^{-} J \psi, \pi^{+} \pi^{-} h_{c}, \pi^{+} \pi^{-} \psi(3686)$ and $\pi^{+} D^{0} D^{*-}$. The parameters are consist with each other within errors. Enhancements around similar positions are observed for $e^{+} e^{-} \rightarrow K^{+} K^{-} J \psi, \eta h_{c}$ and $\gamma \eta_{c}$. Several light hadrons decay channels are investigated to search for $Y(4220)$ and no clear signal is observed.

XVII International Conference on Hadron Spectroscopy and Structure - Hadron2017

25-29 September, 2017

University of Salamanca, Salamanca, Spain

${ }^{*}$ Speaker. 


\section{Introduction}

The famous $Y(4260)$ was first observed by BaBar collaboration [1] in the initial-state-radiation (ISR) process $e^{+} e^{-} \rightarrow \gamma_{\mathrm{ISR}} \pi^{+} \pi^{-} J \psi$ with a mass around $4.26 \mathrm{GeV} / c^{2}$. It was confirmed by CLEO [2] and Belle [3] subsequently using same process. Lately with more statistics [4, 5], the signal of $Y(4260)$ becomes more clear and the mass is tend to be less than $4.26 \mathrm{GeV} / c^{2}$.

While being above the open-charm threshold, the $Y(4260)$ is only observed in the hiddencharm decay channels, not like other conventional vector charmonium. From both the cross section measurements of total hadronic [6] and open-charm [7] processes, there is no enhancement around the peak of $Y(4260)$. While for the cross section measurements of other hidden-charm channels at BESIII, such as $\pi Z_{c}$ [8], $\gamma X(3872)$ [9] and $\omega \chi_{c 0}$ [10], the enhancement is very clear, which indicate that these channels could come from $Y(4260)$ decay. From previous cross section measurements of $e^{+} e^{-} \rightarrow \pi^{+} \pi^{-} J / \psi$, the line shape of $Y(4260)$ is not exactly a symmetric Breit-Wigner (BW). With more data at BESIII, we can measure the cross sections for some hidden and open-charm channels precisely to see if there is any fine structure.

\section{Data at BESIII}

Up to now, BESIII has accumulated about $12 \mathrm{fb}^{-1}$ data above $4.0 \mathrm{GeV}$. We have several large data samples (XYZ sample) before to study the XYZ states and about 100 sub-samples (R-scan data) with luminosity about $7 \mathrm{pb}^{-1}$ for each of them. In additional, 9 data samples (blue dots) have been taken recently for the future study of $Y(4260)$. Since the only known produced way of $Y(4260)$ is $e^{+} e^{-}$annihilation, the BESIII is the ideal place to study the $Y(4260)$.

Recently we performed some analyses to measure the cross sections of some hadronic and radiation hidden-charm transitions, open-charm channels, and some light hadrons channels.

\section{Hadronic transition}

\subsection{Cross section measurements of $e^{+} e^{-} \rightarrow \pi^{+} \pi^{-} J \psi$}

With more data at BESIII, we measured the cross section of $e^{+} e^{-} \rightarrow \pi^{+} \pi^{-} J \psi$ with significantly improved precision [12]. The results are shown in Fig. 1. The cross section is defined as:

$$
\sigma(\sqrt{s})=\frac{N^{\text {sig }}}{\mathscr{L}_{\text {int }}(1+\delta) \varepsilon \mathscr{B}},
$$

where $N^{\text {sig }}$ and $\mathscr{L}_{\text {int }}$ are the number of signal events and integrated luminosity, respectively, $(1+\delta)$ is the ISR correction factor and obtained by iterations, $\varepsilon$ and $\mathscr{B}$ are the detection efficiency and branching ratio for $J / \psi$ to lepton pairs.

From Fig. 1, a shoulder around $4.3 \mathrm{GeV}$ is observed, which indicate that the structure known as $Y(4260)$ is not caused by one resonance but two. We use three coherent BW with the three body phase space, two for the structure around $4.26 \mathrm{GeV}$ and one for the possible $Y(4008)$, and an incoherent $\psi(3770)$ to parameterize the line shape of $\pi^{+} \pi^{-} J \psi$. The fit shows good consistent with data, and the $Y(4260)$ peak can be explained by two resonance, one with mass $4222.0 \pm 3.1 \pm 1.4$ $\mathrm{MeV} / \mathrm{c}^{2}$ and width $44.1 \pm 4.3 \pm 2.0 \mathrm{MeV}$, another one with mass $4320.0 \pm 10.4 \pm 7.0 \mathrm{Mev} / \mathrm{C}^{2}$ and 

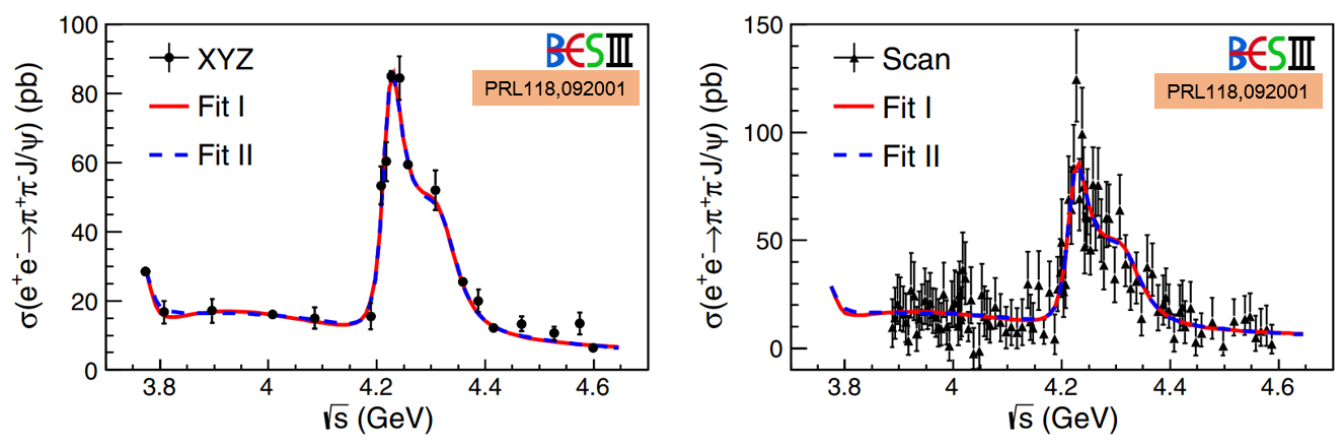

Figure 1: Cross sections of $e^{+} e^{-} \rightarrow \pi^{+} \pi^{-} J \psi$ for XYZ data (left) and R-scan data (right).

width $101.4_{-19.7}^{+25.3} \pm 10.2 \mathrm{MeV}$, respectively. The first resonance agrees with the $Y(4260)$ reported by BaBar, CLEO and Belle, but much narrower thanks to more statistics at BESIII. The statistical significance for the second resonance is larger than $7.6 \sigma$, and the parameters are similar with the $Y(4360)$. For $Y(4008)$, we can not confirm it.

\subsection{Cross section measurements of $e^{+} e^{-} \rightarrow \pi^{+} \pi^{-} h_{c}$}

Like $\pi^{+} \pi^{-} J \psi$, we measured the cross section of $e^{+} e^{-} \rightarrow \pi^{+} \pi^{-} h_{c}$ [13] with same data and the cross section is defined with the same method. For the reconstruction of $h_{c}$, we use the E1 transition $\gamma \eta_{c}$ and use 16 decay channels to tag $\eta_{c}$.

The cross sections are shown in Fig. 2 (left) and obtained by iterations. The cross section goes down at 4.23 and $4.4 \mathrm{GeV}$, which indicate there should be one structure around 4.23 and one around $4.4 \mathrm{GeV}$. Two coherent BW with three body phase space are used to get the parameters. The statistical significance of two structures assumption over one is larger than $10 \sigma$. The first one has mass $4218.4_{-4.5}^{+5.5} \pm 0.9 \mathrm{MeV} / c^{2}$ and width $66.0_{-8.3}^{+12.3} \pm 0.4 \mathrm{MeV}$ and consist with the similar structure observed in $\pi^{+} \pi^{-} J / \psi$ and $\omega \chi_{c} 0$, the second one has mass $4391.5_{-6.8}^{+6.3} \pm 1.0 \mathrm{MeV} / c^{2}$ and width $139.5_{-20.6}^{+16.2} \pm 0.6 \mathrm{MeV}$.
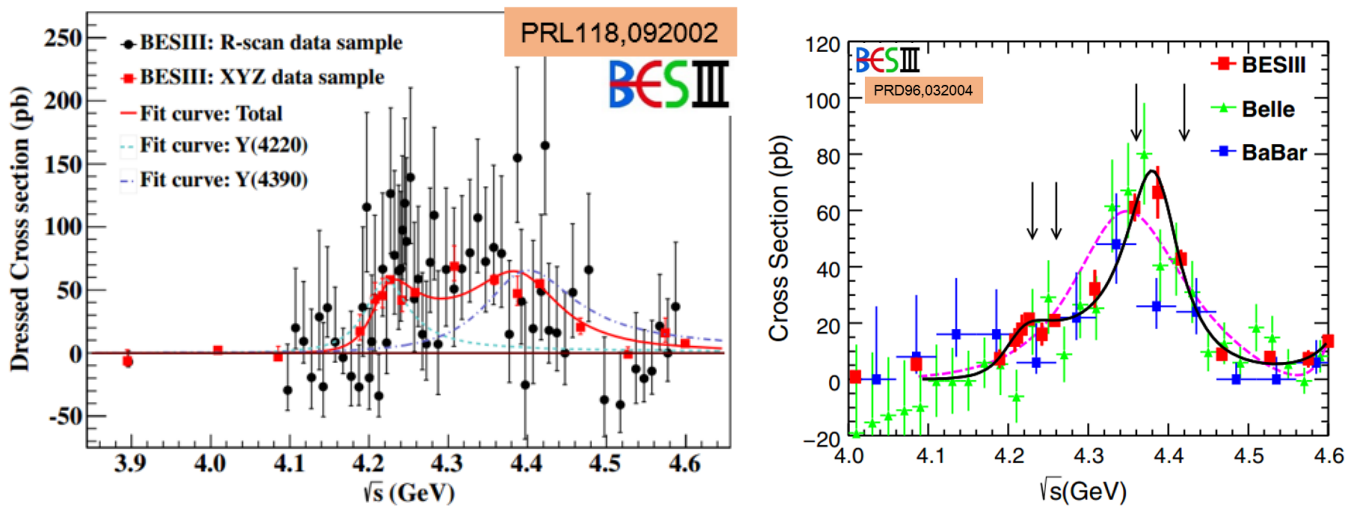

Figure 2: Cross sections of $e^{+} e^{-} \rightarrow \pi^{+} \pi^{-} h_{c}$ (left) and $\pi^{+} \pi^{-} \psi(3686)$ (right) 


\subsection{Cross section measurements of $e^{+} e^{-} \rightarrow \pi^{+} \pi^{-} \psi(3686)$}

We studied the $e^{+} e^{-} \rightarrow \pi^{+} \pi^{-} \psi(3686)$ with $\psi(3686)$ to $\pi^{+} \pi^{-} J \psi$ and $\pi^{0} \pi^{0}\left(\pi^{0} \eta \gamma \gamma\right) J \psi$ [14]. The cross sections are shown in Fig. 2 (right). With more statistics at BESIII, the cross section has much better precision than the results from BaBar [15] and Belle [16]. Besides the $Y(4360)$, there is an enhancement around $4.22 \mathrm{GeV}$, which maybe caused by $Y(4260)$, and it goes up around 4.6 GeV. Three coherent BW are used to parameterize the line shape. The structure around 4.22 $\mathrm{GeV}$ is observed in $\pi^{+} \pi^{-} \psi(3686)$ for the first time with a significance of $5.8 \sigma$, and has a mass $4209.5 \pm 7.4 \pm 1.4 \mathrm{MeV} / c^{2}$ and width $80.1 \pm 24.6 \pm 2.9 \mathrm{MeV}$, which are consist with the structure observed in $\pi^{+} \pi^{-} J \psi$ and $\pi^{+} \pi^{-} h_{c}$. The second structure has a mass $4383.8 \pm 4.2 \pm 0.8 \mathrm{MeV} / c^{2}$ and width $84.2 \pm 12.5 \pm 2.1 \mathrm{MeV}$ and consist with the that in $\pi^{+} \pi^{-} h_{c}$.

\subsection{Cross section measurements of $e^{+} e^{-} \rightarrow K K J \psi$}

The process of $e^{+} e^{-} \rightarrow K K J \psi$ is investigated and the Born cross sections after vacuum polarization correction are shown in Fig. 3 for $K^{+} K^{-} J \psi$ (left) and $K_{S} K_{S} J \psi$ (right). The ratio of cross sections between this two channels are consist with prediction by isospin symmetry, but the ratio between $K K J \psi$ and $\pi^{+} \pi^{-} J \psi$ are not the same along c.m. energy, which indicate more complex structure in $K K J \psi$
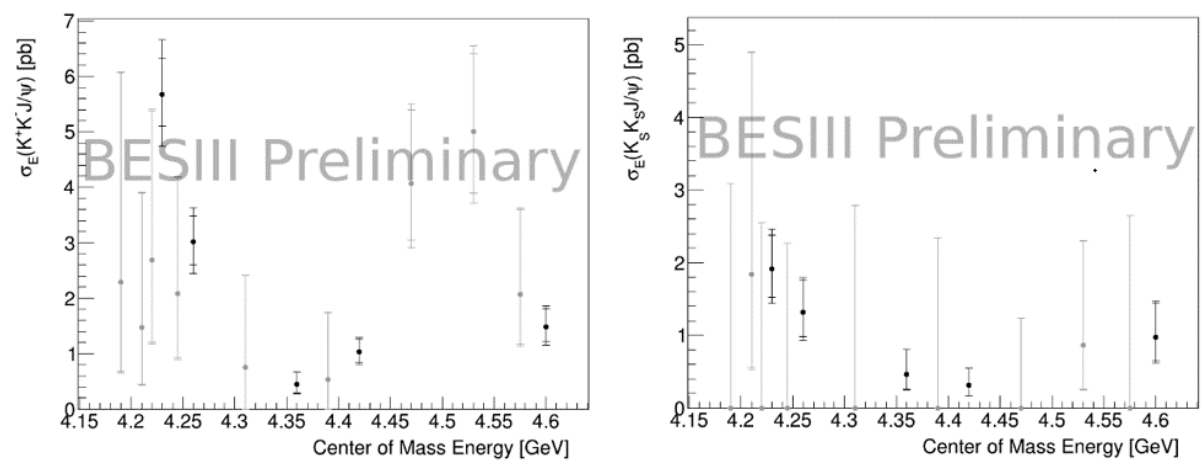

Figure 3: Cross sections of $e^{+} e^{-} \rightarrow K^{+} K^{-} J \psi$ (left) and $K_{S} K_{S} J \psi$ (right)

\subsection{Cross section measurements of $e^{+} e^{-} \rightarrow \eta h_{c}$}

We observe the process $e^{+} e^{-} \rightarrow \eta h_{c}$ for the first time and the Born cross section are shown in Fig. 4 (right). Clear signal is observed at $4.23 \mathrm{GeV}$ and may come from the same structure in $e^{+} e^{-} \rightarrow \eta J \psi$ [17]. The ratio between cross section of $\eta h_{c}$ and $\eta J \psi$ is determined to be $0.20 \pm 0.07$ and $1.79 \pm 0.84$ at 4.23 and $4.36 \mathrm{GeV}$, and larger than theoretical predictions of $\psi(4160)$ and $\psi(4415)$ decays.

\section{Radiative transition: $e^{+} e^{-} \rightarrow \gamma \eta_{c}$}

Besides the hadronic transition, we also use the radiative transition to search for the $Y(4260)$. We observe first evidence for the process $e^{+} e^{-} \rightarrow \gamma \eta_{c}$ between 4.01 and $4.60 \mathrm{GeV}$ [18], and the Born cross section are shown in Fig. 4 (right). The cross section is better explained by assuming $\gamma \eta_{c}$ 

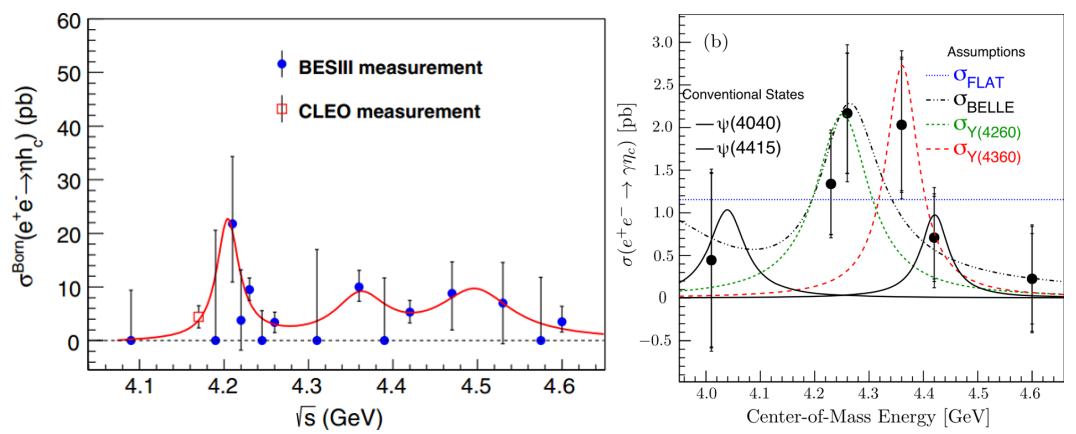

Figure 4: Cross sections of $e^{+} e^{-} \rightarrow \eta h_{c}$ (left) and $\gamma \eta_{c}$ (right)

comes from $Y(4260)$ decays than from conventional charmonium $\psi(4040), \psi(4160)$ and $\psi(4415)$. The enhancement around $4.26 \mathrm{GeV}$ may suggest the existence of a hybrid charmonium state [19].

\section{Open-charm: $e^{+} e^{-} \rightarrow \pi^{+} D^{0} D^{*-}$}

To understand the $Y(4260)$, we perform a study of a open-charm process $e^{+} e^{-} \rightarrow \pi^{+} D^{0} D^{*-}$ since its production could be strongly enhanced above the $D \bar{D}_{1}(2420)$ threshold [20]. Fig. 5 (left) shows the distribution of cross sections. We observe a clear enhancement around $4.23 \mathrm{GeV}$ and a broad one around $4.4 \mathrm{GeV}$. Two coherent BW are used to fit the cross sections. The mass and width for the first structure are $4228.6 \pm 4.1 \pm 5.0 \mathrm{MeV} / c^{2}$ and $77.1 \pm 6.8 \pm 2.7 \mathrm{MeV}$, and for the second one are $4404.6 \pm 7.4 \pm 4.8 \mathrm{MeV} / \mathrm{c}^{2}$ and $191.7 \pm 13.0 \pm 15.1 \mathrm{MeV}$, respectively. The first structure has similar parameters with that in the above processes, and more importantly consist with the prediction of $D \bar{D}_{1}(2420)$ molecule.
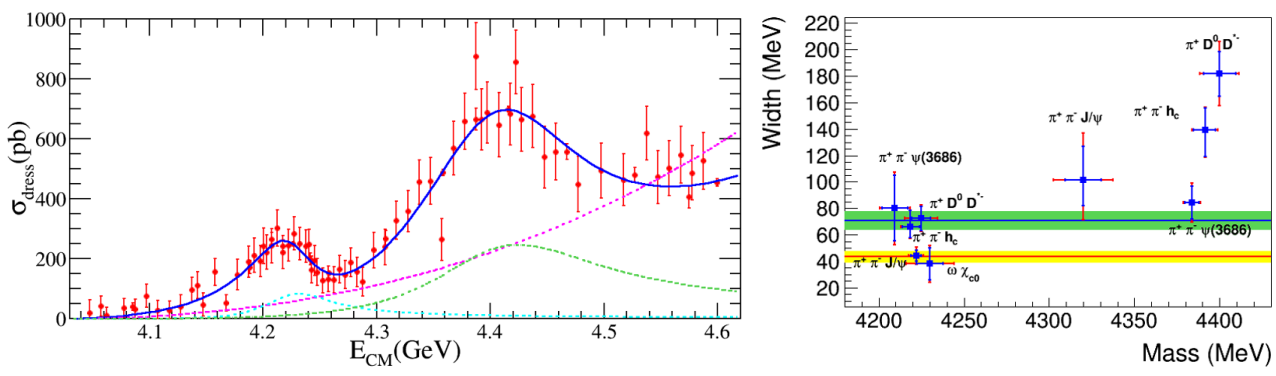

Figure 5: Cross sections of $e^{+} e^{-} \rightarrow \pi^{+} D^{0} D^{*-}$ (left) and parameters for the resonances in this proceeding (right).

\section{Light hadron channels}

In addition, we performed several analysis to search for the light hadron decays of $Y(4260)$. The Born cross section of $e^{+} e^{-} \rightarrow \phi \phi \phi, \phi \phi \omega$ [21], $p \bar{p} \pi^{0}$ [22] and $\eta Y$ (2175) [23] are measured from 4.0 to $4.6 \mathrm{GeV}$. No obvious $Y(4260)$ signal was observed. 


\section{Summary and discussion}

In summary, we observed a structure with mass around $4220 \mathrm{MeV}$, denoted as $Y(4220)$, in $e^{+} e^{-} \rightarrow \pi^{+} \pi^{-} J \psi, \pi^{+} \pi^{-} h_{c}, \pi^{+} \pi^{-} \psi(3686)$ and open-charm channel $\pi^{+} D^{0} D^{*-}$, similar with that in $\omega \chi_{c 0}$. Fig. 5 (right) shows the parameters, the masses are consist well between different channels, while the width measurements have larger difference and maybe caused by other effects, e.g. open-charm threshold, three body phase space. For the $\eta h_{c}$ and $\gamma \eta_{c}$, enhancement around the peak of $Y(4220)$ is observed and we need more data to confirm. No light hadron decays of $Y(4220)$ was observed. With more data at BESIII, we can investigate more channels and the nature of $Y(4220)$ will become more and more clear.

\section{References}

[1] B. Aubert et al. (BaBar Collaboration), Phys. Rev. Lett. 95, 142001 (2005).

[2] Q. He et al. (CLEO Collaboration), Phys. Rev. D 74, 091104(R) (2006).

[3] C. Z. Yuan et al. (Belle Collaboration), Phys. Rev. Lett. 99, 182004 (2007).

[4] J. P. Lees et al. (BaBar Collaboration), Phys. Rev. D 86, 051102 (2012).

[5] Z. Q. Liu et al. (Belle Collaboration), Phys. Rev. Lett. 110, 252002 (2013).

[6] C. Patrignani et al. (Particle Data Group), Chin. Phys. C 40, 100001 (2016).

[7] W. M. Song, Ph.D. Thesis, IHEP, 2015.

[8] M. Ablikim et al. (BESIII Collaboration), Phys. Rev. Lett. 115, 112003 (2015).

[9] M. Ablikim et al. (BESIII Collaboration), Phys. Rev. Lett. 112, 092001 (2014).

[10] M. Ablikim et al. (BESIII Collaboration), Phys. Rev. Lett. 114, 092003 (2015).

[11] M. Ablikim et al. (BESIII Collaboration), Nucl. Instrum. Meth. A 614, 345 (2010).

[12] M. Ablikim et al. (BESIII Collaboration), Phys. Rev. Lett. 118, 092001 (2017).

[13] M. Ablikim et al. (BESIII Collaboration), Phys. Rev. Lett. 118, 092002 (2017).

[14] M. Ablikim et al. (BESIII Collaboration), Phys. Rev. D 96, 032004 (2017).

[15] J. P. Lees et al. (BaBar Collaboration), Phys. Rev. D 89, 111103 (2014).

[16] X. L. Wang et al. (Belle Collaboration), Phys. Rev. D 91, 112007 (2015).

[17] M. Ablikim et al. (BESIII Collaboration), Phys. Rev. D 91, 112005 (2015).

[18] M. Ablikim et al. (BESIII Collaboration), Phys. Rev. D 96, 051101 (2017).

[19] J. J. Dudek, R. G. Edwards and C. E. Thomas, Phys. Rev. D 79, 094504 (2009).

[20] W, Qin, S. R. Xue, and Q. Zhao, Phys. Rev. D 94, 054035 (2016).

[21] M. Ablikim et al. (BESIII Collaboration), Phys. Lett. B 774, 78 (2017).

[22] M. Ablikim et al. (BESIII Collaboration), Phys. Lett. B 771, 45 (2017).

[23] M. Ablikim et al. (BESIII Collaboration), arXiv: 1709.04323. 